\title{
The First Case of New Variant COVID-19 Originating in the United Kingdom Detected in a Returning Student - Shanghai Municipality, China, December 14, 2020
}

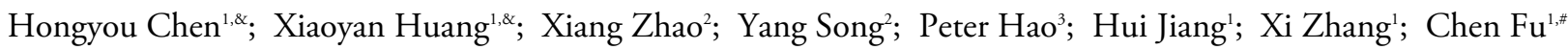

On December 14, 2020, a 23-year-old female returning from the United Kingdom (UK) via airplane was tested by the laboratory of Shanghai Customs using nose swab to test for coronavirus disease 2019 (COVID-19). At 20:30 on December 14, Shanghai CDC received notification from the Shanghai Customs that the patient tested positive for COVID-19. By 22:00, the patient was transported by ambulance from the isolation point to the fever clinic of Jiading District Central Hospital. Due to travel history from the UK and abnormalities in nucleic acid test results, the hospital organized a consultation with experts and formed a recommendation to transfer the patient to Fudan University's Public Health Clinical Center for further diagnosis and treatment, which was carried out the following day. On December 15, Jiading District $\mathrm{CDC}$ retested using a nasopharyngeal swab sample and the nucleic acid result was positive for COVID-19.

An epidemiological investigation revealed that the patient had a negative COVID-19 test result on December 12, 2020, two days before her flight to return to China. According to her statement, she had no exposure to symptomatic individuals and had not purchased or been exposed to frozen food products or raw meat. While remaining in the UK, the patient described running in a nearby park without wearing a mask and taking off her mask to eat and drink while waiting to board the plane. These are all potential situations for exposure, especially with 1.86 million confirmed cases of COVID-19 having been reported in the UK as of December 15, 2020. At the time of case report, the patient was diagnosed as a mild case according to the epidemiological investigation, symptoms and laboratory test.

On December 24, the sample of the COVID-19 patient was sequenced using MGI MGISEQ-200 platform (Sequence ID: NC20SCU2740-1). This strain was dissimilar with the previous Shanghai strain detected in the Shanghai outbreak in November, suggesting different route of transmission (1) (Figure 1). Compared with the Wuhan reference sequence (EPI_ISL_ 402119) (2), this new Shanghai strain showed 32 nucleotide variation sites, containing the single nucleotide polymorphisms (SNPs) that defined L-lineage European branch I. This Shanghai strain shared all 28 variation sites (C241T, C913T, C3037T, C3267T, C5388A, C5986T, T6954C, C14408T, C14676T, C15279T, T16176C, A23063T, C23271A, A23403G, C23604A, C23709T, T24506G, G24914C, C27972T, G28048T, A28111G, G28280C, A28281T, T28282A, G28881A, G28882A, G28883C, and C28977T) that were first detected in the VUI202012/01 (Pangolin lineage B.1.1.7) variant from the UK (3-4). It also had 2 specific variation sites (C3177T, A28271del), from which site C3177T was also detected in the VUI202012/01 variants that has been circulating since late October in the UK (Figure 1). Just like the other VUI202012/01 variants, the Shanghai strain also had 3 amino acid deletions (H69del, V70del and Y144del) and 7 amino acid mutations (N501Y, A570D, D614G, P681H, T716I, S982A and D1118H) in the spike protein. The Shanghai strain NC20SCU2740-1 is the first imported VUI202012/01 variant in China and poses a great potential threat to the prevention and control of COVID-19 in China.

Several control measures have been implemented in the response to this case. The patient has been transferred to the designated medical institution for isolation and treatment. Due to the closed-loop management upon passenger's arrival at the airport, close contact investigation has been initiated according to national and municipal work plans: 1) close contacts currently include passengers with seats in the same row, passengers within 3 rows of seats of the patient, and flight attendants providing cabin services to that section; 2) medical personnel at the point of isolation and at the central hospital are not deemed closed contacts due to the effective personal protective 

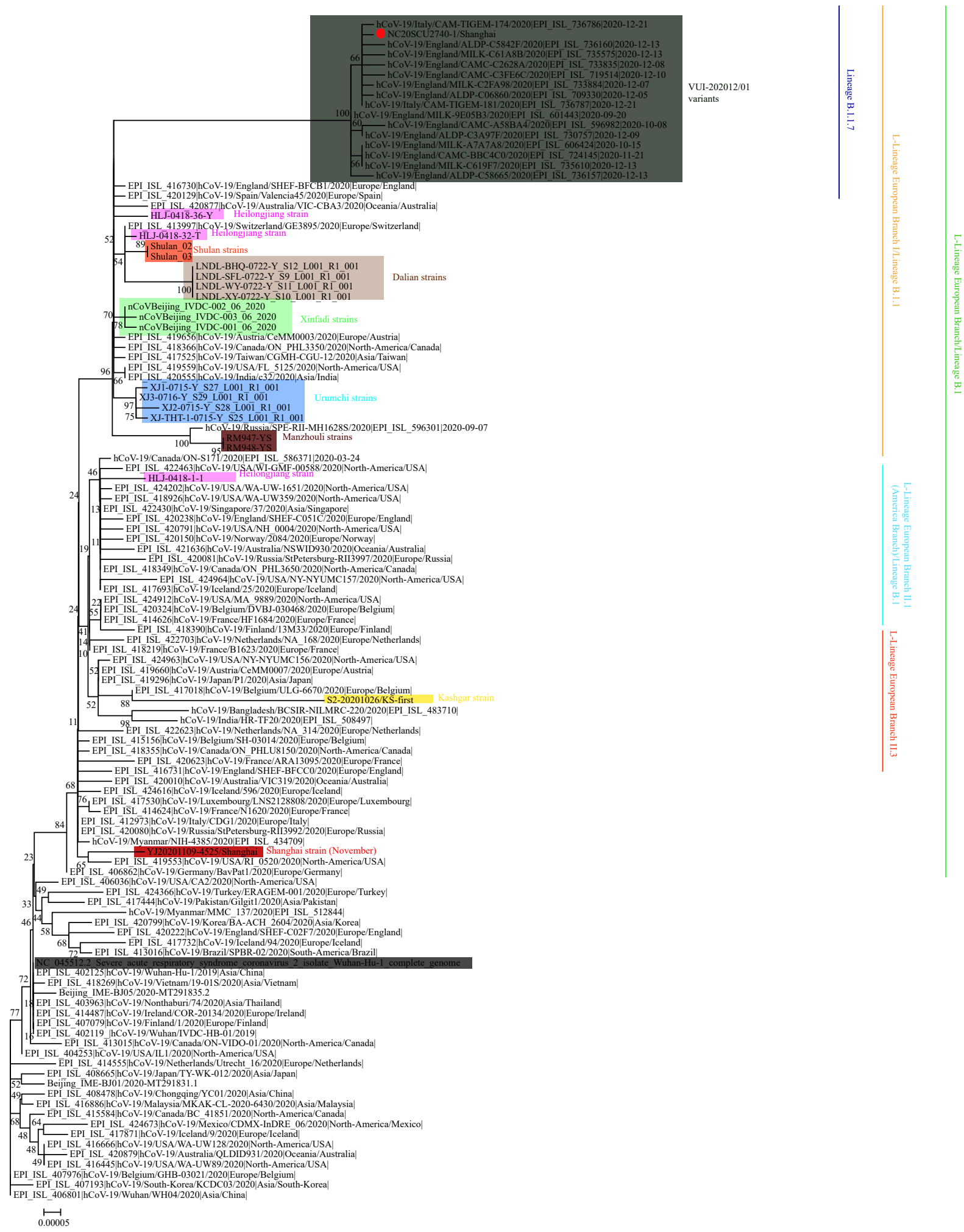

FIGURE 1. Phylogenetic tree based on the full-length genome sequences of the COVID-19 virus.

The VUI-202012/01 variants are highlighted in dark green and the first imported VUI-202012/01 variant of China detected in Shanghai is indicated by a red dot. The strains associated with specific outbreaks in China are as follows: Manzhouli City (burgundy/darker red); Shanghai Municipality in November (red); Kashgar Prefecture (yellow); Urumchi City (blue); Beijing Municipality Xinfadi Wholesale Market (light green); northeastern China including Heilongjiang Province (pink) and Shulan (orange) related to imported cases; Dalian City (brown); Wuhan City in December 2019 (dark gray). The S(A)- or L(B)lineage and sublineages of the COVID-19 virus were marked and colored on the right. 
measures; and 3) other possible close contacts are being investigated. Specific venues associated with the patient are being comprehensively disinfected. Experts have been assembled to supervise relevant work including disinfection, personal protection, and nosocomial infection control in areas in the medical facilities.

doi: $10.46234 / \mathrm{ccdcw} 2020.270$

\# Corresponding author: Chen Fu, fuchen@scdc.sh.cn.

${ }^{1}$ Shanghai Municipal Center for Disease Control and Prevention, Shanghai, China; ${ }^{2}$ National Institute for Viral Disease Control and Prevention, China CDC, Beijing, China; ${ }^{3}$ Chinese Center for Disease Control and Prevention, Beijing, China.

${ }^{\&}$ Joint first authors.

Submitted: December 28, 2020; Accepted: December 29, 2020

\section{REFERENCES}

1. Fang FH, Song Y, Hao LP, Nie K, Sun XD. A case of COVID-19 detected in a cargo worker at Pudong Airport - Shanghai Municipality, China, November 8, 2020. China CDC Wkly 2020;2(47):910. http://dx.doi.org/10.46234/ccdcw2020.246.

2. Wu F, Zhao S, Yu B, Chen YM, Wang W, Song ZG, et al. A new coronavirus associated with human respiratory disease in China. Nature 2020;579(7798):265. http://dx.doi.org/10.1038/s41586-020-2008-3.

3. Rambaut A, Holmes EC, O’Toole Á, Hill V, McCrone JT, Ruis C, et al. A dynamic nomenclature proposal for SARS-CoV-2 lineages to assistgenomic epidemiology. Nat Microbiol 2020. http://dx.doi.org/ 10.1038/s41564-020-0770-5.2.

4. European Centre for Disease Prevention and Control, Rapid increase of a SARS-CoV-2 variant with multiple spike protein mutations observed in the United Kingdom- 20 December 2020. ECDC: Stockholm; 2020. https://www.ecdc.europa.eu/sites/default/files/documents/SARS-CoV-2variant-multiple-spike-protein-mutations-United-Kingdom.pdf. 